http://www.e-journals.net

\title{
Synthesis and Antibacterial Activity of Chalcones and Pyrimidine-2-ones
}

\author{
A. K. PAREKH* and K. K. DESAI \\ Department of chemistry, \\ Veer Narmad South Gujarat University, \\ Surat-395007, India.
}

Received 27 December 2004; Accepted 9 March 2005

\begin{abstract}
Some new chalcones have been prepared by Claisen-schmidt condensation of ketone and different aromatic aldehydes. These chalcones on condensation with urea in presence of acid gave Pyrimidine-2-ones. The synthesized compounds have been characterized by elemental analysis, IR and ${ }^{1} \mathrm{H}$ NMR spectral data. They have been screened for their antibacterial activity against Gram positive bacteria B. subtillis \& S. aureus and Gram negative bacteria E. coli \& S. typhi.
\end{abstract}

Key words: Chalcones, Pyrimidine-2-ones, Antibacterial activity.

\section{Introduction}

The chemistry of chalcones have been recognized as a significant field of study. The chalcones and their derivatives possess good antibacterial activity. ${ }^{1-3}$ Besides this they show antifungal ${ }^{4}$, insecticidal ${ }^{5}$, anaesthetic $^{6}$, anti-inflammatory, analgesic and ulcerogenics ${ }^{7}$ properties. Pyrimidines and their derivatives are considered to be important as drugs and agricultural chemicals. Some substituted pyrimidines and their derivatives have been reported to possess interesting biological activities such as hypnotic properties ${ }^{8}$, antifungal ${ }^{9}$, antitumour ${ }^{10}$, activities. Many pyrimidines are used as thyroid drugs. ${ }^{11}$

\section{Experimental}

Melting points of synthesized compounds have been taken in open capillaries and are uncorrected. Purity of compounds were checked by TLC on silica gel coated glass plates. IR spectra were recorded by preparing pallets in $\mathrm{KBr}$ on Shimatzu FT-IR 8300 spectrophotometer. ${ }^{1} \mathrm{H}$ NMR spectra recorded on "Perkin Elmer Model -32 NMR spectrometer" of $300 \mathrm{MHz}$ using TMS as an internal standard. All the synthesized compounds have been screened for their antibacterial activity. 


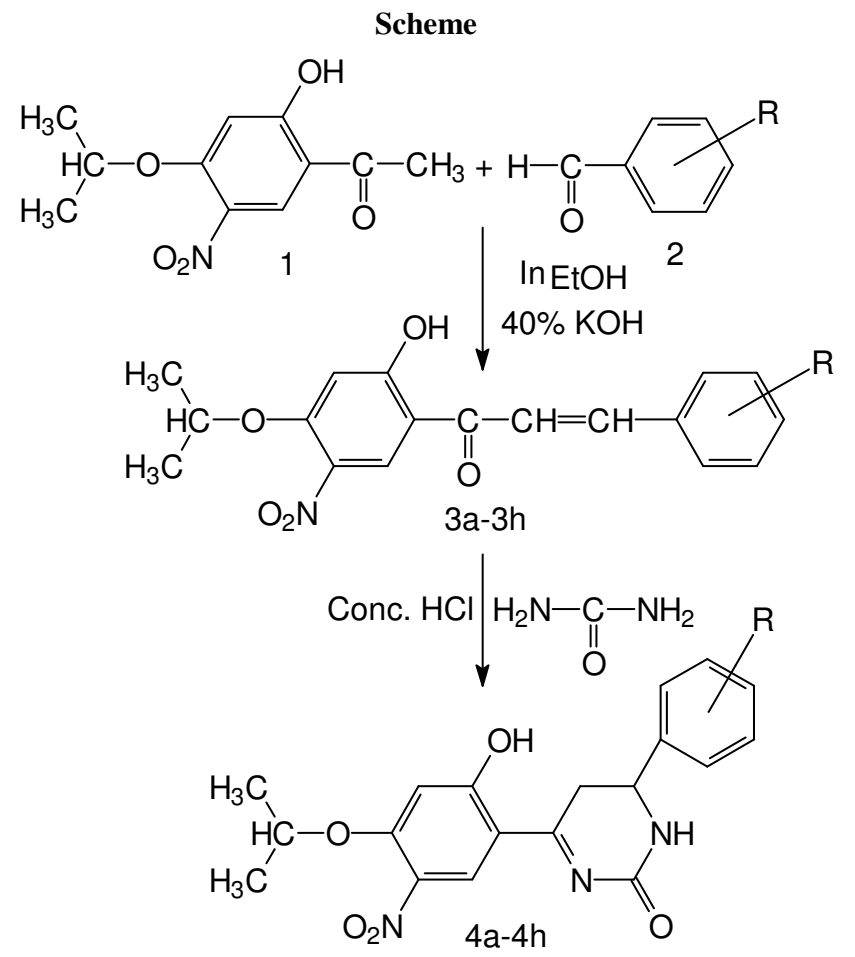

Preparation of chalcones

2-Hydroxy-4-isopropoxy-5-nitro-acetophenone $(0.01 \mathrm{~mole})$ dissolved in ethanol and various substituted aromatic aldehydes $(0.01 \mathrm{~mole})$ dissolved in ethanol were mixed and potassium hydroxide solution $(40 \%, 25 \mathrm{ml})$ was added to it drop wise with stirring and kept overnight at room temperature. The content was then poured over crushed ice and acidified with cold dilute $\mathrm{HCl}(10 \%)$. The solid thus separated was filtered, washed with water, dried and crystallised from acetic acid.

Preparation of 4-[2'-hydroxy-4'-isopropoxy-5'-nitrophen-1'-yl]-6-[substituted aryl]-tetrahydropyrimidine-2-one.

A mixture of different 2 '-hydroxy-4'-isopropoxy-5'-nitro substituted chalcones $(0.01$ mole $)$ and urea $(0.01 \mathrm{~mole})$ in ethanol $(40 \mathrm{ml})$ and concentrated $\mathrm{HCl}(10 \mathrm{ml})$ were refluxed at $70-80^{\circ} \mathrm{C}$ for 16 hours. The reaction mixture was then cooled and poured into ice water with stirring. The product thus separated was filtered, washed with water and crystallised from ethanol.

\section{Results and Discussion}

Structures of the compounds synthesized have been confirmed by elemental analysis, IR spectra and ${ }^{1} \mathrm{H}$ NMR spectra.

2'-Hydroxy-4'-isopropoxy-5'-nitrosubstituted chalcones show IR absorption bands at 3438$3466 \mathrm{~cm}^{-1}$ (-OHstr.), 3050-3062 cm-1 (Aromatic C-H str.), $1093 \mathrm{~cm}^{-1}$ (C-O-C str.), 1540-1555 cm (N=O str.), $1633-1640 \mathrm{~cm}^{-1}(\mathrm{C}=\mathrm{O}$ str. $), 1583-1600 \mathrm{~cm}^{-1}(\mathrm{C}=\mathrm{C}$ str. $)$ and $1380-1390 \mathrm{~cm}^{-1}(\mathrm{C}-\mathrm{H}$ ben. $)$ and NMR signals at $\delta=1.25\left(6 \mathrm{H},(\mathrm{d}),-\mathrm{CH}\left[\mathrm{CH}_{3}\right]_{2}\right), \delta=2.32\left(1 \mathrm{H},(\mathrm{m}),-\mathrm{CH}\left[\mathrm{CH}_{3}\right]_{2}\right), \delta=7.05(\alpha \mathrm{H},(\mathrm{d}),-\mathrm{COCH}=\mathrm{CH})$, $\delta=7.79(\beta \mathrm{H},(\mathrm{d}),-\mathrm{COCH}=\mathrm{CH}), \delta=9.17(1 \mathrm{H},(\mathrm{s}), \mathrm{Ar}-\mathrm{OH}), \delta=6.70-8.23(6 \mathrm{H},(\mathrm{m}), \mathrm{Ar}-\mathrm{H})$.

Pyrimidine-2-ones show IR absorption bands at $3422-3475 \mathrm{~cm}^{-1}$ (-OH str.), $3225-3260 \mathrm{~cm}^{-1}(\mathrm{~N}-\mathrm{H}$ str.) $3067-3090 \mathrm{~cm}^{-1}$ (Aromatic C-H str.), $1075-1108 \mathrm{~cm}^{-1}\left(\mathrm{C}-\mathrm{O}-\mathrm{C}\right.$ str.), $1540-1550 \mathrm{~cm}^{-1}(\mathrm{~N}=\mathrm{O}$ str.), $1650-1670 \mathrm{~cm}^{-1}(\mathrm{C}=\mathrm{O}$ str. $), 1593 \mathrm{~cm}^{-1}(\mathrm{C}=\mathrm{N}$ str. $)$ and $1380-1390 \mathrm{~cm}^{-1}$ (C-H ben.) and NMR Signals at $\delta=1.29\left(6 \mathrm{H},(\mathrm{d}),-\mathrm{CH}\left[\mathrm{CH}_{3}\right]_{2}\right), \delta=2.17\left(1 \mathrm{H},(\mathrm{m}),-\mathrm{CH}\left[\mathrm{CH}_{3}\right]_{2}\right), \delta=2.73(1 \mathrm{H},(\mathrm{t}),-\mathrm{CH}$ of pyrimidine ring $)$, 
$\delta=3.11(1 \mathrm{H},(\mathrm{d}),-\mathrm{CH}$ of pyrimidine ring $), \delta=4.05(1 \mathrm{H},(\mathrm{d}),-\mathrm{NH}), \delta=9.01(1 \mathrm{H},(\mathrm{s}), \mathrm{Ar}-\mathrm{OH}), \delta=6.75-7.40$ $(6 \mathrm{H},(\mathrm{m}), \mathrm{Ar}-\mathrm{H})$.

Table-1 Characterization Data of Compounds 3a-3h

\begin{tabular}{|c|c|c|c|c|c|c|c|c|}
\hline \multirow{2}{*}{ No. } & \multirow{2}{*}{$\mathrm{R}$} & \multirow{2}{*}{ M.F. } & \multirow{2}{*}{ M.W. } & \multirow{2}{*}{$\begin{array}{c}\text { Yield } \\
(\%)\end{array}$} & \multirow{2}{*}{$\begin{array}{l}\text { M.P. } \\
\left({ }^{0} \mathrm{C}\right)\end{array}$} & \multicolumn{3}{|c|}{$\begin{array}{c}\% \text { Analysis } \\
\text { Calc.(Found) }\end{array}$} \\
\hline & & & & & & $\mathrm{C}$ & $\mathrm{H}$ & $\mathrm{N}$ \\
\hline $3 a$ & $\mathrm{H}$ & $\mathrm{C}_{18} \mathrm{H}_{17} \mathrm{O}_{5} \mathrm{~N}$ & 327 & 69 & 182 & $\begin{array}{c}66.05 \\
(66.01)\end{array}$ & $\begin{array}{c}5.19 \\
(5.07)\end{array}$ & $\begin{array}{c}4.28 \\
(4.19)\end{array}$ \\
\hline $3 b$ & $2-\mathrm{Cl}$ & $\mathrm{C}_{18} \mathrm{H}_{16} \mathrm{O}_{5} \mathrm{NCl}$ & 361.5 & 67 & 191 & $\begin{array}{c}59.75 \\
(59.69)\end{array}$ & $\begin{array}{c}4.42 \\
(4.34)\end{array}$ & $\begin{array}{c}3.87 \\
(3.80)\end{array}$ \\
\hline $3 c$ & $4-\mathrm{Cl}$ & $\mathrm{C}_{18} \mathrm{H}_{16} \mathrm{O}_{5} \mathrm{NCl}$ & 361.5 & 74 & 207 & $\begin{array}{c}59.75 \\
(59.70)\end{array}$ & $\begin{array}{c}4.42 \\
(4.31)\end{array}$ & $\begin{array}{c}3.87 \\
(3.78)\end{array}$ \\
\hline $3 \mathrm{~d}$ & $2,4-(\mathrm{Cl})_{2}$ & $\mathrm{C}_{18} \mathrm{H}_{15} \mathrm{O}_{5} \mathrm{NCl}_{2}$ & 396 & 76 & 223 & $\begin{array}{c}54.54 \\
(54.48)\end{array}$ & $\begin{array}{c}3.78 \\
(3.66)\end{array}$ & $\begin{array}{c}3.53 \\
(3.45)\end{array}$ \\
\hline $3 e$ & $2-\mathrm{OCH}_{3}$ & $\mathrm{C}_{19} \mathrm{H}_{19} \mathrm{O}_{6} \mathrm{~N}$ & 357 & 65 & 216 & $\begin{array}{c}63.86 \\
(63.72)\end{array}$ & $\begin{array}{c}5.32 \\
(5.27)\end{array}$ & $\begin{array}{c}3.92 \\
(3.83)\end{array}$ \\
\hline $3 \mathrm{f}$ & $4-\mathrm{OCH}_{3}$ & $\mathrm{C}_{19} \mathrm{H}_{19} \mathrm{O}_{6} \mathrm{~N}$ & 357 & 68 & 228 & $\begin{array}{c}63.86 \\
(63.74)\end{array}$ & $\begin{array}{c}5.32 \\
(5.26)\end{array}$ & $\begin{array}{c}3.92 \\
(3.81)\end{array}$ \\
\hline $3 g$ & $3-\mathrm{OC}_{6} \mathrm{H}_{5}$ & $\mathrm{C}_{24} \mathrm{H}_{21} \mathrm{O}_{6} \mathrm{~N}$ & 419 & 72 & 252 & $\begin{array}{c}68.73 \\
(68.59)\end{array}$ & $\begin{array}{c}5.01 \\
(4.93)\end{array}$ & $\begin{array}{c}3.34 \\
(3.25)\end{array}$ \\
\hline $3 \mathrm{~h}$ & $4\left[\mathrm{~N}\left(\mathrm{CH}_{3}\right)_{2}\right]$ & $\mathrm{C}_{20} \mathrm{H}_{22} \mathrm{O}_{5} \mathrm{~N}_{2}$ & 419 & 75 & 232 & $\begin{array}{c}64.88 \\
(64.72) \\
\end{array}$ & $\begin{array}{c}5.94 \\
(5.86) \\
\end{array}$ & $\begin{array}{r}7.56 \\
(7.44) \\
\end{array}$ \\
\hline
\end{tabular}

$\underline{\text { Table-2 Characterization data of compounds } 4 \mathrm{a}-4 \mathrm{~h}}$

\begin{tabular}{|c|c|c|c|c|c|c|c|c|}
\hline \multirow[t]{2}{*}{ No. } & \multirow[t]{2}{*}{$\mathrm{R}$} & \multirow[t]{2}{*}{ M.F. } & \multirow[t]{2}{*}{ M.W. } & \multirow{2}{*}{$\begin{array}{l}\text { Yield } \\
(\%)\end{array}$} & \multirow{2}{*}{$\begin{array}{l}\text { M.P. } \\
\left({ }^{0} \mathrm{C}\right)\end{array}$} & \multicolumn{3}{|c|}{$\begin{array}{c}\text { \% Analysis } \\
\text { Calc.(Found) }\end{array}$} \\
\hline & & & & & & $\mathrm{C}$ & $\mathrm{H}$ & $\mathrm{N}$ \\
\hline $4 a$ & $\mathrm{H}$ & $\mathrm{C}_{19} \mathrm{H}_{19} \mathrm{O}_{5} \mathrm{~N}_{3}$ & 369 & 67 & 133 & $\begin{array}{c}61.78 \\
(61.72)\end{array}$ & $\begin{array}{c}5.14 \\
(5.13)\end{array}$ & $\begin{array}{c}11.38 \\
(11.35)\end{array}$ \\
\hline $4 \mathrm{~b}$ & $2-\mathrm{Cl}$ & $\mathrm{C}_{19} \mathrm{H}_{18} \mathrm{O}_{5} \mathrm{~N}_{3} \mathrm{Cl}$ & 303.5 & 69 & 139 & $\begin{array}{c}56.50 \\
(56.44)\end{array}$ & $\begin{array}{c}4.46 \\
(4.41)\end{array}$ & $\begin{array}{c}10.40 \\
(10.36)\end{array}$ \\
\hline $4 c$ & $4-\mathrm{Cl}$ & $\mathrm{C}_{19} \mathrm{H}_{18} \mathrm{O}_{5} \mathrm{~N}_{3} \mathrm{Cl}$ & 303.5 & 73 & 144 & $\begin{array}{c}56.50 \\
(56.48)\end{array}$ & $\begin{array}{c}4.46 \\
(4.40)\end{array}$ & $\begin{array}{c}10.40 \\
(10.32)\end{array}$ \\
\hline $4 d$ & $2,4-(\mathrm{Cl})_{2}$ & $\mathrm{C}_{19} \mathrm{H}_{17} \mathrm{O}_{5} \mathrm{~N}_{3} \mathrm{Cl}_{2}$ & 438 & 76 & 157 & $\begin{array}{c}52.05 \\
(52.01)\end{array}$ & $\begin{array}{c}3.88 \\
(3.84)\end{array}$ & $\begin{array}{c}9.58 \\
(9.51)\end{array}$ \\
\hline $4 \mathrm{e}$ & $2-\mathrm{OCH}_{3}$ & $\mathrm{C}_{20} \mathrm{H}_{21} \mathrm{O}_{6} \mathrm{~N}_{3}$ & 399 & 71 & 152 & $\begin{array}{c}60.15 \\
(60.09)\end{array}$ & $\begin{array}{c}5.26 \\
(5.23)\end{array}$ & $\begin{array}{c}10.52 \\
(10.44)\end{array}$ \\
\hline $4 \mathrm{f}$ & $4-\mathrm{OCH}_{3}$ & $\mathrm{C}_{20} \mathrm{H}_{21} \mathrm{O}_{6} \mathrm{~N}_{3}$ & 399 & 74 & 163 & $\begin{array}{c}60.15 \\
(60.07)\end{array}$ & $\begin{array}{c}5.26 \\
(5.21)\end{array}$ & $\begin{array}{c}10.52 \\
(10.48)\end{array}$ \\
\hline $4 g$ & $3-\mathrm{OC}_{6} \mathrm{H}_{5}$ & $\mathrm{C}_{25} \mathrm{H}_{23} \mathrm{O}_{6} \mathrm{~N}_{3}$ & 461 & 73 & 179 & $\begin{array}{c}65.07 \\
(65.04)\end{array}$ & $\begin{array}{c}4.98 \\
(4.95)\end{array}$ & $\begin{array}{c}9.11 \\
(9.07)\end{array}$ \\
\hline $4 \mathrm{~h}$ & $4-\left[\mathrm{N}\left(\mathrm{CH}_{3}\right)_{2}\right]$ & $\mathrm{C}_{21} \mathrm{H}_{24} \mathrm{O}_{5} \mathrm{~N}_{4}$ & 412 & 76 & 168 & $\begin{array}{c}61.16 \\
(61.12)\end{array}$ & $\begin{array}{c}5.82 \\
(5.77)\end{array}$ & $\begin{array}{c}13.59 \\
(13.54)\end{array}$ \\
\hline
\end{tabular}

\section{Antibacterial activity}

The synthesized compounds were tested for their antibacterial activity by measuring the inhibition area on agar plates (diffusimetric method) ${ }^{12}$ with Gram positive bacteria B. subtillis \& S. aureus and Gram negative bacteria E. coli \& $\mathrm{S}$ typhi as test germs.

The results of antibacterial screening indicated that very good activity was shown by compound $3 \mathrm{~d}$ against all four organisms while compound $3 \mathrm{~g}$ was found to possess moderate activity against Gram negative bacteria but poor activity against Gram positive bacteria. Compounds $4 \mathrm{~d}$, $4 \mathrm{f}$ and $4 \mathrm{~h}$ exhibited 
very good activity. Other compounds showed moderate activity against both Gram positive and Gram negative bacteria.

112 A. K. PAREKH et al.,

Table-3 Antibacterial Activity of Newly Synthesized Compounds, Zone of Inhibition (mm).

\begin{tabular}{ccccc}
\hline \multirow{2}{*}{ No. } & \multicolumn{2}{c}{ Gram positive } & \multicolumn{2}{c}{ Gram negative } \\
\cline { 2 - 5 } & B. Subtillis & S. aureus & E. coli & S. typhi \\
\hline 3a & 6 & 8 & 9 & 4 \\
3b & 8 & 7 & 9 & 7 \\
3c & 12 & 10 & 10 & 11 \\
3d & 15 & 17 & 16 & 14 \\
3e & 6 & 8 & 7 & 8 \\
3f & 8 & 7 & 8 & 6 \\
3g & 8 & 7 & 12 & 12 \\
$3 \mathrm{~h}$ & 8 & 14 & 12 & 7 \\
4a & 7 & 6 & 6 & 4 \\
$4 \mathrm{~b}$ & 8 & 6 & 8 & 7 \\
$4 \mathrm{c}$ & 9 & 11 & - & 8 \\
4d & 14 & 16 & 12 & 7 \\
$4 \mathrm{e}$ & - & 11 & 13 & 7 \\
$4 \mathrm{f}$ & 15 & 16 & 12 & 6 \\
4g & 9 & 8 & 8 & 14 \\
\hline $\mathrm{h}$ & 17 & 18 & 18 & \\
\hline
\end{tabular}

\section{Acknowledgement}

The authors are thankful to South Gujarat University, Surat for providing research facilities and CDRI, Lucknow, for providing ${ }^{1} \mathrm{H}$ NMR spectra.

\section{References}

1. Desai M D and Desai K K, Asian J. Chem. 2002, 14(2), 974.

2. Desai M D and Desai K K, Asian J. Chem. 2002, 14(2), 995.

3. Patel H S, Patel V K and Dixit B C, Oriental J. Chem. 2001, 17(3), 411.

4. Mehta K J, Patel V S and Parikh A R, J. Indian Chem. Soc. 1978, 50, 241.

5. Mudaliar V and Joshi V, Indian J. Chem. 1995, 34B, 456.

6. Hosni G and Saad S F, Acta Chim. Acad. Sci. Hung. 1995, 86, 263. Chem. Abstr.; 1979, 84, 30959w.

7. Hishmat O H, El-Diwani H I and Melek F R, Indian J. Chem. 1996, 35B, 30.

8. Dickey J B and Gary A R, Org. Synth. Coll. 1943, 2, 62.

9. El-Hashash M A, Mahmoud M R and Madboli S A, Indian J. Chem. 1993, 32B, 449.

10. Lyttle D A and Petering H G, J. Am. Chem. Soc. 1958, 80, 6459.

11. Liberti P and Stanbury J B, Annu. Rev. Pharmacol. 1971, 11, 113. 


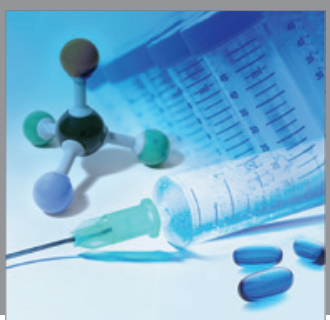

International Journal of

Medicinal Chemistry

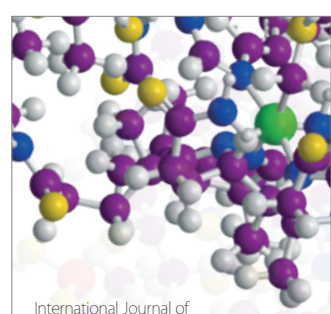

Carbohydrate Chemistry

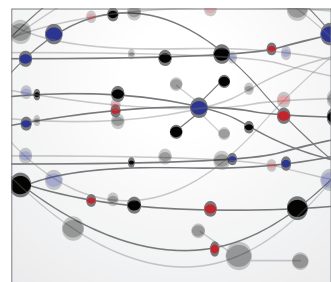

The Scientific World Journal
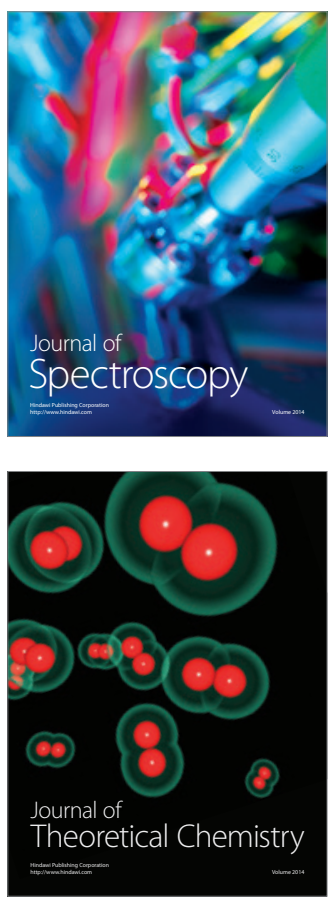
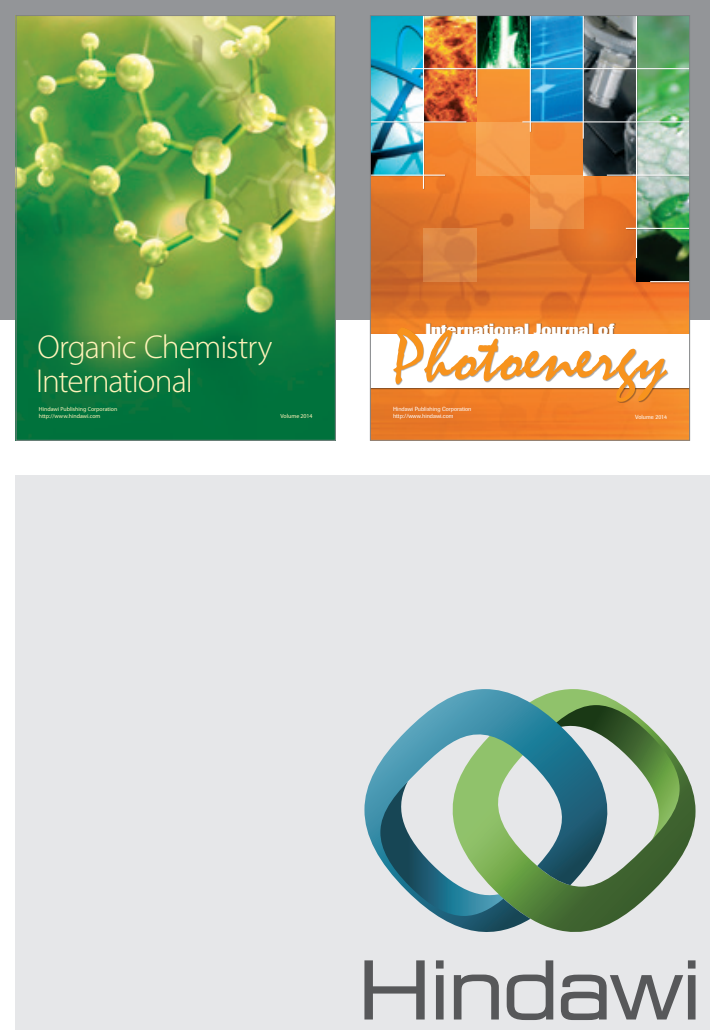

Submit your manuscripts at

http://www.hindawi.com
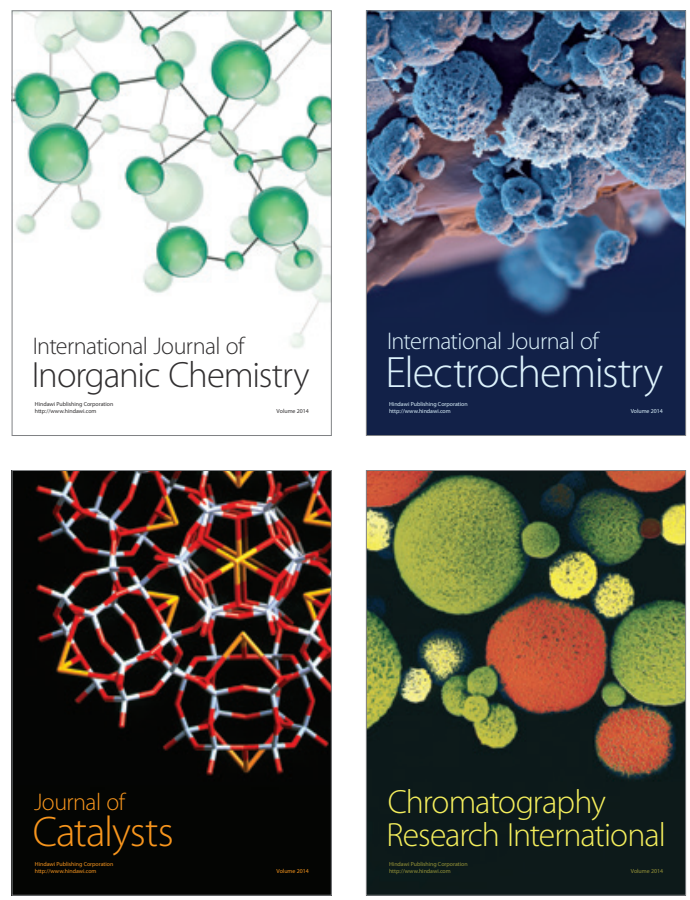
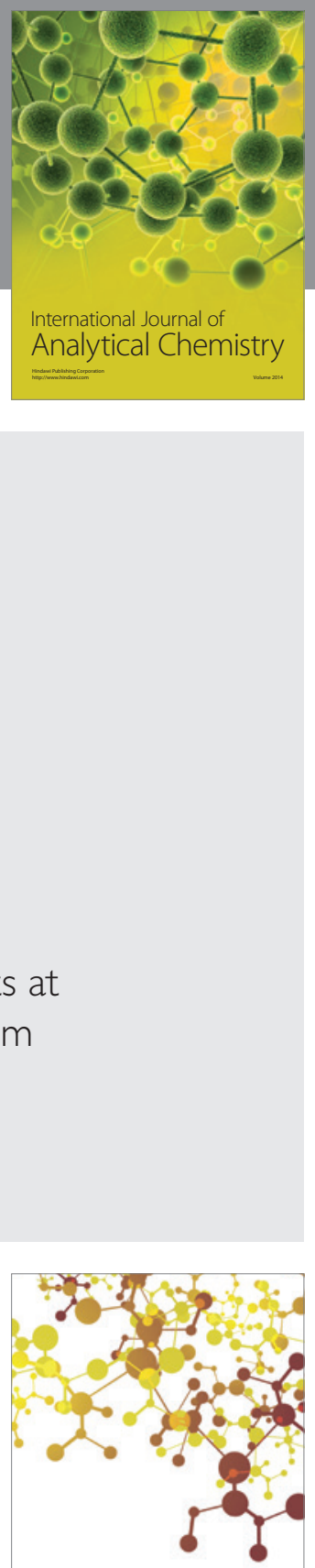

Journal of

Applied Chemistry
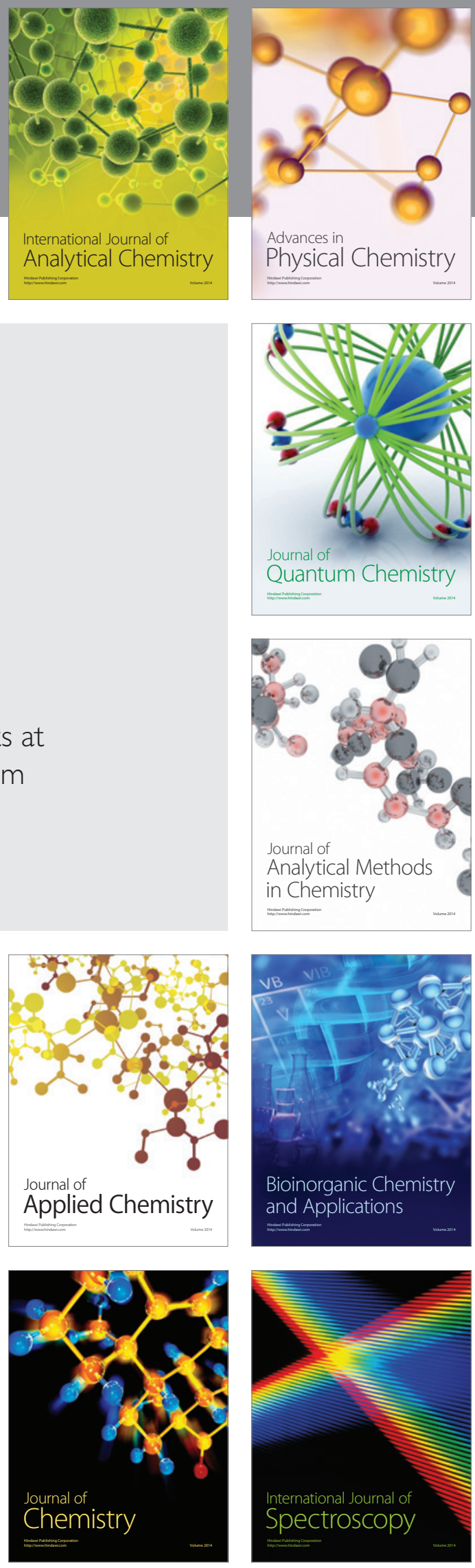